\title{
基于短周期地震仪内置GPS位置数据的东南极 冰流速测定
}

傅䂞 ${ }^{1,2,3}$, 郭井学 ${ }^{4}$, 陈晓非 $1,2,3^{*}$

1. 深圳市深远海油气勘探技术重点实验室(南方科技大学), 深圳 518055;

2. 南方科技大学地球与空间科学系, 深圳 518055 ;

3. 南方海洋科学与工程广东省实验室(广州), 广州 511458 ;

4. 中国极地研究中心极地科学重点实验室, 上海 200136

* 通讯作者, E-mail: chenxf@sustech.edu.cn

收稿日期: 2021-01-21; 收修改稿日期: 2021-03-08; 接受日期: 2021-03-30; 网络版发表日期: 2021-07-07

国家自然科学基金项目(批准号: 41974044、U1901602、41790465、41876227)和深圳市科技计划项目(编号: KQTD20170810111725321)资助

\begin{abstract}
摘要 冰川表面流速是冰川和冰盖的基本特征, 冰流速的测定对于南北极冰盖物质平衡估算至关重要. 常见的冰 流速测定方法包括采用花杆、雪坑及现场GPS地面测量以及遥感测量法. 地面GPS测量技术具有高精度优点，然 而目前南极大陆冰盖地面GPS监测点覆盖较少; 基于遥感测量技术的冰盖表面流速测量其分辨率和精度较低. 短 周期地震仪内置的导航级GPS接收器记录的位置数据, 尽管其精度不如大地测量GPS的位置精度, 但是可以通过 在足够长的时间内采集的导航级GPS数据来准确测量冰流速. 本文利用中国第36次南极科学考察期间被动源地 震观测时获得的导航级GPS位置信息, 准确的对东南极拉斯曼丘陵冰盖和泰山站区冰盖运动特征进行了追踪. 结 果表明, 两地的冰盖基本都向西偏北运动, 且月均位移大小约 $1 \mathrm{~m}$; 本文在泰山站区的结果与通过大地测量GPS结 果基本吻合, 说明利用短周期地震仪内置GPS位置信息进行冰盖运动特征的追踪是可行的. 靠近中山站的拉斯曼 丘陵局部冰流复杂, 本文获得的间距 $200 \mathrm{~m}$ 的测量结果有助于认识该冰流. 总之, 本文结果对于冰盖动力学研究、 冰川质量平衡研究和在地震成像中评估由冰盖流动带来的系统误差具有重要意义.
\end{abstract}

关键词短周期地震仪, 南极冰盖, 冰流速, GPS

\section{1 引言}

全球气候变暖正在加速南北极冰盖质量亏损 $(\mathrm{Op}-$ penheimer, 1998; Chylek等, 2004; Hanna等, 2005, 2008). 近几十年来, 空气(Straneo和Heimbach, 2013)和 海洋(Fettweis等, 2017)温度显著上升, 格陵兰岛周围的 夏季云层(Hofer等, 2017)减少; 这些变化导致了地表径
流(Trusel等，2018)、冰川上湖泊的形成(Leeson等, 2015)和排水(Palmer等, 2015), 冰山崩塌(Nick等, 2012), 冰川终点撤退(Joughin等，2008). Holland等 (2008) 通过研究北大西洋大气环流及格陵兰Jakobshavn Isbræ冰川的变化，表明其质量亏损加速是由西 海岸地下海洋温度升高触发的. 格陵兰、南极半岛和 西南极部分地区以中等程度(约 $1 \mathrm{~mm} \mathrm{a}^{-1}$ 海平面上升当 
量)进行着质量损失(Hanna等, 2013), 其中格陵兰岛的 冰流失在全球海平面上升中占主要贡献，模型预测表 明在气候变暖的大趋势中，格陵兰岛的质量损失将持 续下去(Pattyn等, 2018). Shepherd等(2018)将体积、流 量和重力变化的卫星观测结果与冰盖表面质量平衡模 型相结合, 显示出南极冰盖在1992 2017年之间损失了 $(27200 \pm 13900)$ 亿吨冰，相当于平均海平面升高 $(7.6$ $\pm 3.9) \mathrm{mm}$.

冰川表面流速是冰川和冰盖的基本特征，它表征 从内陆向海岸区域运输冰的速率、运冰通道及冰块如 何随时间演变等(Rignot等, 2011). 冰流速的测定对于 南北极冰盖物质平衡估算至关重要，传统的冰流速测 定方法包括实地测量和遥感测量. 实地测量通常采用 花杆、雪坑及现场GPS测量为主. Dorrer等(1969)在横 跨南极罗斯冰架的 $910 \mathrm{~km}$ 长的测线上使用了 103 个花 杆来研究冰盖速度场变化, 结果显示麦克默多冰架和 罗斯冰架之间的速度快速增加，冰架中间均匀且几乎 平行的运动. 徐绍铨等 (1988)在中国第二次 (1985/ 1986)和第三次(1986/1987)南极科学考察期间利用花 杆, 在南极半岛纳尔逊岛冰川布设了 6 个冰川运动监测 点, 结合光学经纬仪, 测定了各点的平面位置及高程, 得出了该部冰川以年均 $14.6 \mathrm{~m}$ 的速度向大海流去的结 论. 花杆测量具有操作简单、测量故障率低等优点, 然 而花杆实地重复测量时间间隔较长，且对后勤保障要 求极高. 全球定位系统(Global Positioning System, GPS)测量技术具有全天候、低成本、全方位、高效 率、高精度等优点, 可以十分便捷地在南极地区进行 冰川表面流速的快速测量与实时监测(陈军, 2016). Manson等(2000)在1988 1995年期间, 沿着兰伯特冰川 (Lambert Glacier)流域 $2500 \mathrm{~m}$ 等高线, 采集了 73 个点的 GPS数据, 结果表明在冰川出口位置, 沿着GPS点位路 线, 其冰流速变化在0.5 $63 \mathrm{~m} \mathrm{a}^{-1}$ 之间. 国际横穿南极科 学考察(International Trans-Antarctic Scientific Expedition, ITASE)期间，中国在中山站至Dome-A断面进行 了 7 个地面GPS点复测, 通过对三期复测的观测数据进 行高精度GPS静态定位处理可知, 考察沿线的GPS点 以8 $24 \mathrm{~m} \mathrm{a}^{-1}$ 的速度向西北方向(冰盖边缘方向)流动, 越接近冰盖边缘, 运动速度越快, 最快达到 $100 \mathrm{~m} \mathrm{a}^{-1}$; 同时, 由于冰盖的流动, 引起了垂直方向 $0.2 \sim 1 \mathrm{~m} \mathrm{a}^{-1}$ 的 沉降速度(王清华等，2001).Zhang等(2008)利用 1996 2006年期间，在中山站至Dome-A断面观测的19
个 GPS 重复观测数据, 通过解算认为断面上冰流速由 内陆向海岸逐渐增大; Dome-A区域冰流速小于 $10 \mathrm{~m} \mathrm{a}^{-1}$; 冰原地区冰流速为 $8 \sim 24 \mathrm{~m} \mathrm{a}^{-1}$, 某些局部地区 冰流速达到 $98.2 \mathrm{~m} \mathrm{a}^{-1}$; 冰流方向大致垂直于冰盖表面 高程轮廓, 主要朝向兰伯特冰川盆地(Lambert Glacier Basin).

地面花杆和GPS测量仅能获取局部区域的离散观 测数据, 无法获得大面积的冰川速度场; 基于光学遥感 影像或微波遥感数据的遥感测量方法为获取整个南极 冰盖表面流速场提供了新的技术手段. 光学遥感测量 主要利用图像特征跟踪和相关性计算原理, 通过特征 匹配来计算冰川的流动速度(Scambos等，1992；Frezzotti等, 1998; 陈军, 2016). Heid和Kääb(2012)针对全 球 5 个特征冰川的Landsat光学影像图像, 比较分析了 6 种不同的特征匹配算法, 认为定向图像的互相关 (CCFO)和相位相关算法(COSI-CORR) 是两个最有效的全 球冰川流速监测的匹配方法. 光学遥感易受极地极 夜、太阳辐射、云雾及影像过饱和及匹配算法等约 束, 其精度相对较低. 合成孔径雷达(SAR)采用主动式 的微波成像模式, 可以实现全天时、全天候的对地观 测, 在冰川运动速度提取中占据重要的地位, 是目前 冰川运动速度提取的主要手段. Goldstein等(1993)基 于InSAR数据的干涉相干性估算南极冰盖的冰川表面 流速. Pattyn和Derauw(2002)通过对两个复杂SAR图像 的小图像核进行匹配，计算了Shirase冰川的表面速度 场, Shirase流域下游部分的冰川接近平衡, 显示出轻 微的负失衡. Rignot(2008)利用相控阵合成孔径雷达 (PALSAR)L波段数据计算了西南极阿蒙森海附近冰川 流速，结果表明1996 2007年间，派恩岛冰川(Pine Island Glacier)和史密斯冰川(Smith Glacier)分别加速了 $42 \%$ 和 $83 \%$; 而Thwaites冰川并未加速，而是随着时间 的推移而扩大, 其东部冰架移动速度也翻了 1 倍. 周春 霞等(2014)利用ERS-1/2 tandem数据, 提取并探讨了中 山站附近极记录冰川(Polar Record Glacier)和达尔克 冰川(Dalker Glacier)冰流的运动规律, 结果表明内陆 地区冰流速随季节和年际变化不是非常明显, DInSAR提取监测点冰流速和实测值非常吻合. 周春霞等 (2015)利用 35 天时间间隔的Envisat ASAR数据对, 采 用DInSAR和偏移量跟踪方法提取了格罗夫山(Grove Mountain)地区冰流运动信息.

基于遥感测量方法的冰流速其精度不如GPS地面 
观测结果. 例如, 基于InSAR的冰流速图, 其标称的误 差范围为 $1 \sim 17 \mathrm{~m} \mathrm{a}^{-1}$ (Rignot等, 2011); 而单点GPS的水 平误差小于 $0.1 \mathrm{~m} \mathrm{a}^{-1}$ (Zhang等, 2008). 对于地形平坦的 内陆冰原区域, 由于缺少明显的地表特征, 此外其冰流 速年均变化小于 $10 \mathrm{~m} \mathrm{a}^{-1}$, 此时基于InSAR的遥感测量 方法可能无效(An等, 2015). 因此, 内陆冰盖地面GPS 测量对于南极大陆冰盖流速研究必不可少.

地震仪器一般内置一个导航级单模GPS接收器, 台站在运行过程中, 记录地震数据的同时, 在固定的时 间间隔记录台站的位置信息. 宽频带地震仪器一般每 小时(或每天)记录台站的位置信息, 而短周期地震仪 往往以更短的时间间隔(例如每 $10 \mathrm{~min}$ )记录台站的位 置信息. 尽管这些单模GPS接收器的数据精度不如大 地测量GPS的位置精度, 仍然可以通过在足够长的时 间内采集的单模GPS数据来测量冰流速(An等, 2015). Den Ouden等(2010)通过单模GPS接收机对斯瓦尔巴 特群岛的冰盖流速进行研究. An等(2015)利用中山站 至Dome-A断面以及西南极部分宽频带地震仪记录的 GPS位置追踪冰盖的流动速度. 其研究指出当冰盖的 年均流速大于 $1 \mathrm{~m} \mathrm{a}^{-1}$ 的时候, 通过几个月的导航级单 模GPS位置数据可以获得可靠的冰盖流动速度信息.

中国第36次南极科学考察期间, 我们利用拐角频 率为 $0.2 \mathrm{~Hz}$ 的 100 台短周期地震仪在东南极拉斯曼丘陵 冰盖(中国南极中山站)进行了约一个月的背景噪声地 震学观测, 此外使用 4 个短周期地震仪在泰山站进行了 一个月的观测; 在记录地震数据的同时每个台站记录 了 4000多个GPS位置数据. 本文利用短周期地震仪的 副产品(GPS位置数据), 进行了地震测线区域冰盖流 速矢量场的估算, 该结果对于冰盖动力学研究、冰川 质量平衡研究以及地震成像中由于冰盖流动带来的系 统性误差评估具有重要意义.

\section{2 数据采集}

中国第36次南极科学考察(CHINARE36, 2019/ 2020)期间, 我们利用 100 台短周期地震仪在东南极拉 斯曼丘陵冰盖布设了一条地震测线. 图 $1 \mathrm{a}$ 为东南极冰 盖表面等高线图, 其高程从沿海中山站向内陆Dome$\mathrm{A}$ 逐渐递增到 $4 \mathrm{~km}$. 中山站 $\left(69.3734^{\circ} \mathrm{S}, 76.3779^{\circ} \mathrm{E}\right)$ 为常 年考察站, 位于拉斯曼丘陵出露基岩上; 距离中山站 $520 \mathrm{~km}$ 的夏季考察站泰山站 $\left(73.8658^{\circ} \mathrm{S}, 76.9829^{\circ} \mathrm{E}\right)$ 冰
面高程约为 $2621 \mathrm{~m}$; 夏季考察站昆仑站 $\left(80.4200^{\circ} \mathrm{S}\right.$, $77.1130^{\circ} \mathrm{E}$ )位于Dome-A冰穹, 其冰面高程为 $4087 \mathrm{~m}$. 图 中黑色箭头代表利用1997 2005年期间地面GPS观测 获取的各测点冰流速方向和大小, 可见接近Dome-A区 域, 其年均冰盖流动大小接近 $1 \mathrm{~m} \mathrm{a}^{-1}$; 靠近海边的局部 区域冰盖流动接近 $100 \mathrm{~m} \mathrm{a}^{-1}$; 而冰盖大部分区域冰流 速大小在8 $24 \mathrm{~m} \mathrm{a}^{-1}$; 且冰盖主流方向为西偏北(Zhang 等, 2008). 图1b所示红色三角形代表地震台站, 台间距 为 $200 \mathrm{~m}$; 其中第一个台站距离中山站约 $8 \mathrm{~km}$, 其所在 冰面高程约为 $200 \mathrm{~m}$; 最后一个台站距离中山站的直线 距离约 $25 \mathrm{~km}$, 所在冰面高程约为 $650 \mathrm{~m}$. 该测线东侧为 达尔克冰川, 地形起伏较大, 其冰舌前端以每年 $100 \mathrm{~m} \mathrm{a}^{-1}$ 的速度流入普里兹湾(Prydz Bay). 第一条测 线观测结束后, 我们将台站251(图1b黄色三角形)布设 于中山站熊猫码头基岩上进行了一个月的观测. 图 $1 \mathrm{c}$ 所示为在泰山站布设的地震台站, 其所处位置地势比 较平坦; 台站288位于泰山站站址位置, 其他 3 台地震仪 等间隔分布于半径为 $1.67 \mathrm{~km}$ 的同心圆. 此次实验中使 用的短周期地震仪为Smartsolo-BD3C-5, 内置Ublox$7 \mathrm{Q}$ 单模GPS接收器, 其精度为 $2 \mathrm{~m}$. 地震仪采集地震波 形数据的同时, 每隔10min记录台站的GPS位置信息.

\section{3 数据处理}

地震资料分析过程中往往假设地震台站的位置 是相对固定的. 如果不考虑冰盖(或冰川)的运动, 则地 震成像中可能会存在系统性误差, 因此有必要利用地 震仪记录的GPS位置数据对冰盖运动进行追踪. 地震 数据采集期间连续记录的GPS位置数据, 其经度和纬 度分别为 $\Lambda(t)$ 和 $\phi(t)$. 首先按一定时间间隔(例如 5 天) 将一个月的原始 $\mathrm{GPS}$ 位置数据分为 $K$ 段, 各段的经纬 度分别为 $\Lambda_{k}(t)$ 和 $\phi_{k}(t)$, 每一段经纬度理论上均满足正 态分布. 偏离均值 2.5 倍标准差的经纬度不满足正态 分布, 记为异常点, 需要剔除. 例如, 台站 251 位于基 岩上, 记录的GPS经纬度分别如图 $2 \mathrm{a}$ 和 $2 \mathrm{~b}$ 所示, 黑色虚 椭圆中为不满足正态分布的异常点, 首先进行剔除处 理. 其中红色直方图代表2020年1月1日至2020年1月5 日的GPS位置数据; 而绿色直方图代表 2020 年 1 月 26 日至 2020 年 1 月 30 日的GPS位置数据. 可以发现当台 站位于基岩时，不管是经度还是纬度，其分布特征随 着时间推移基本没有变化. 反观图 $2 \mathrm{c}$ 和 $2 \mathrm{~d}$, 图中显示 


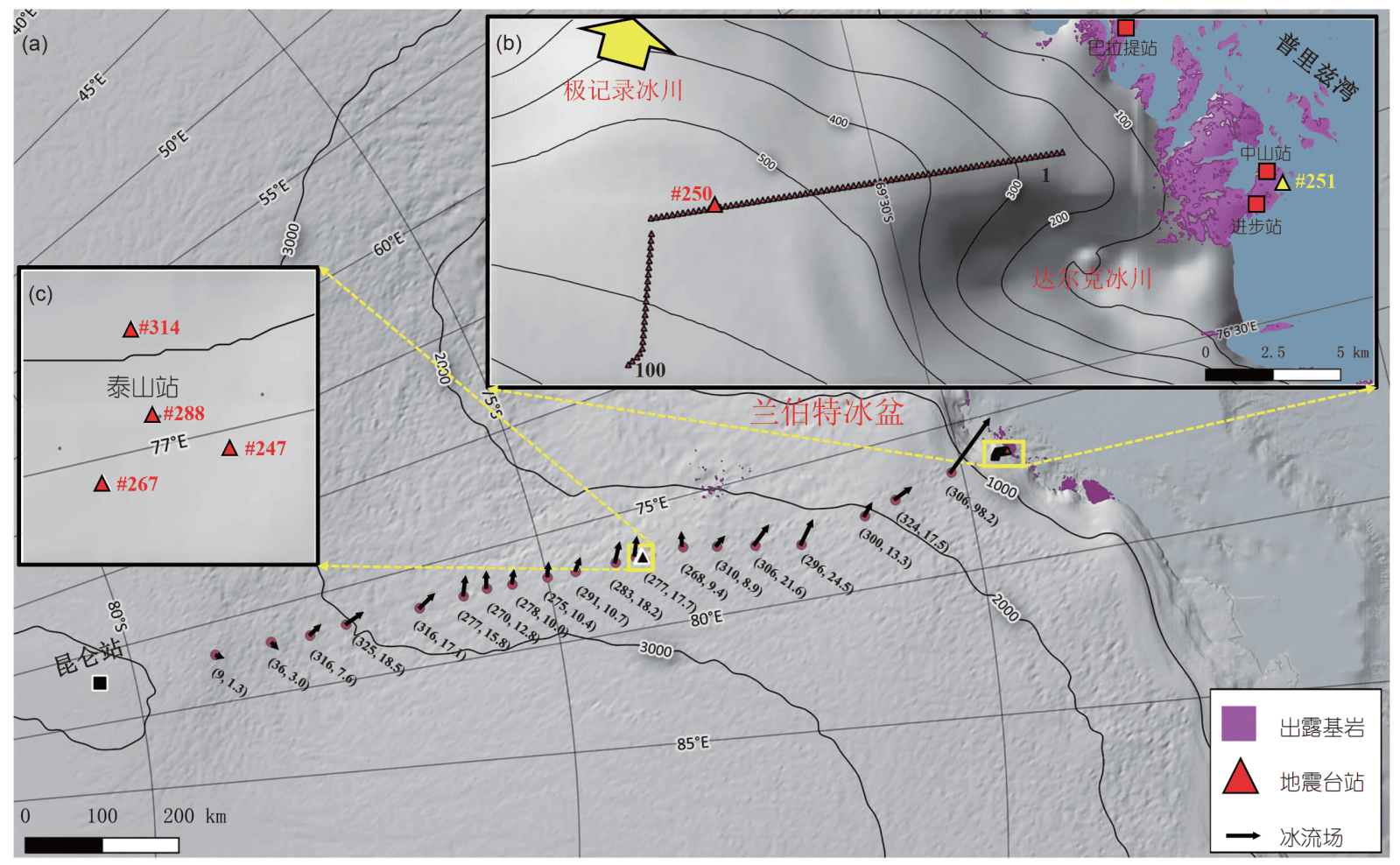

图 1 短周期密集线性台阵位置图

(a) 东南极冰盖表面等高线图, 黑色箭头代表中山站至昆仑站断面大地测量GPS测点处冰流速方向和年均速率, 引自Zhang等(2008), 中国第36 次南极科学考察期间(2019/2020), 短周期地震仪器(红色三角形)位于中山站附近和泰山站. (b) 中山站附近拉斯曼丘陵冰盖, 地震测线东北方 向为达尔克冰川, 测线西侧为极记录冰川, 测线北端为普里兹湾, 红色方框分别为俄罗斯进步站、中国中山站及印度巴拉提站, 红色台站\# 250 的观测时间为2019/12/06 2019/12/27, 而黄色台站\#251的观测时间为2020/01/01 2020/01/30. (c) 泰山站区冰盖, 台站\#288位于泰山站站址. 图 中紫色代表出露基岩, 黑色矢量箭头代表冰盖流动方向和速率

的是台站 250 位于拉斯曼丘陵冰盖期间记录的GPS位 置数据，其中红色直方图代表2019年12月6日至2019 年12月10日期间的数据; 而绿色直方图代表2019年12 月 23 日至2019年12月27日期间的数据. 可以发现, 当 台站位于冰盖时, 其经度分布特征, 随着时间推移发 生了整体偏移; 而纬度分布特征仅发生了微小的变化. 说明随着时间的推移，在冰盖上的台站 250 整体向西 移动了.

为了量化冰盖运动方向和位移大小，对第一个时 间段的平均经纬度分别记为 $\bar{\phi}$, 和 $\bar{\lambda}_{1}$; 第 $K$ 段的平均经纬 度分别记为 $\bar{\phi}_{k}$ 和 $\bar{\lambda}_{k}$. 经过坐标变换后, 第一段和第 $K \mathbf{E}^{-}$ 对应的大地坐标分别为 $\left(X_{1}, Y_{1}\right)$ 和 $\left(X_{2}, Y_{2}\right)$, 对应的冰盖 运动方向和位移分别为

$\theta=\arctan \left(\frac{Y_{2}-Y_{1}}{X_{2}-X_{1}}\right)$

$$
s=\sqrt{\left(X_{2}-X_{1}\right)^{2}+\left(Y_{2}-Y_{1}\right)^{2}} .
$$

当地震台站251位于基岩时, 将其记录的GPS位置 数据按 6 天时间间隔分为 $5 \mathbf{E}^{-}$. 图 $3 \mathrm{a}$ 中不同时间段的 GPS位置坐标分别用红、绿、蓝、黄和青色表示, 各 段对应的平均值用大圆圈表示; 理论上，当台站位于 基岩时, 随着时间推移其位移量为零; 而图3a中观测 到其最大位移约为 $0.2 \mathrm{~m}$, 故而通过短周期地震仪内置 单模GPS接收机的位置数据来追踪冰盖流动的误差大 约为 $0.2 \mathrm{~m} \mathrm{mon}^{-1}$. 图 $3 \mathrm{~b}$ 为地震台站 250 位于拉斯曼丘陵 冰盖时不同时间段GPS位置坐标，可见随着时间推移， 其平均位置整体向西移动了约 $1 \mathrm{~m}$.

\section{4 结果与讨论}

对所有台站的GPS位置数据按等间隔分为若干 

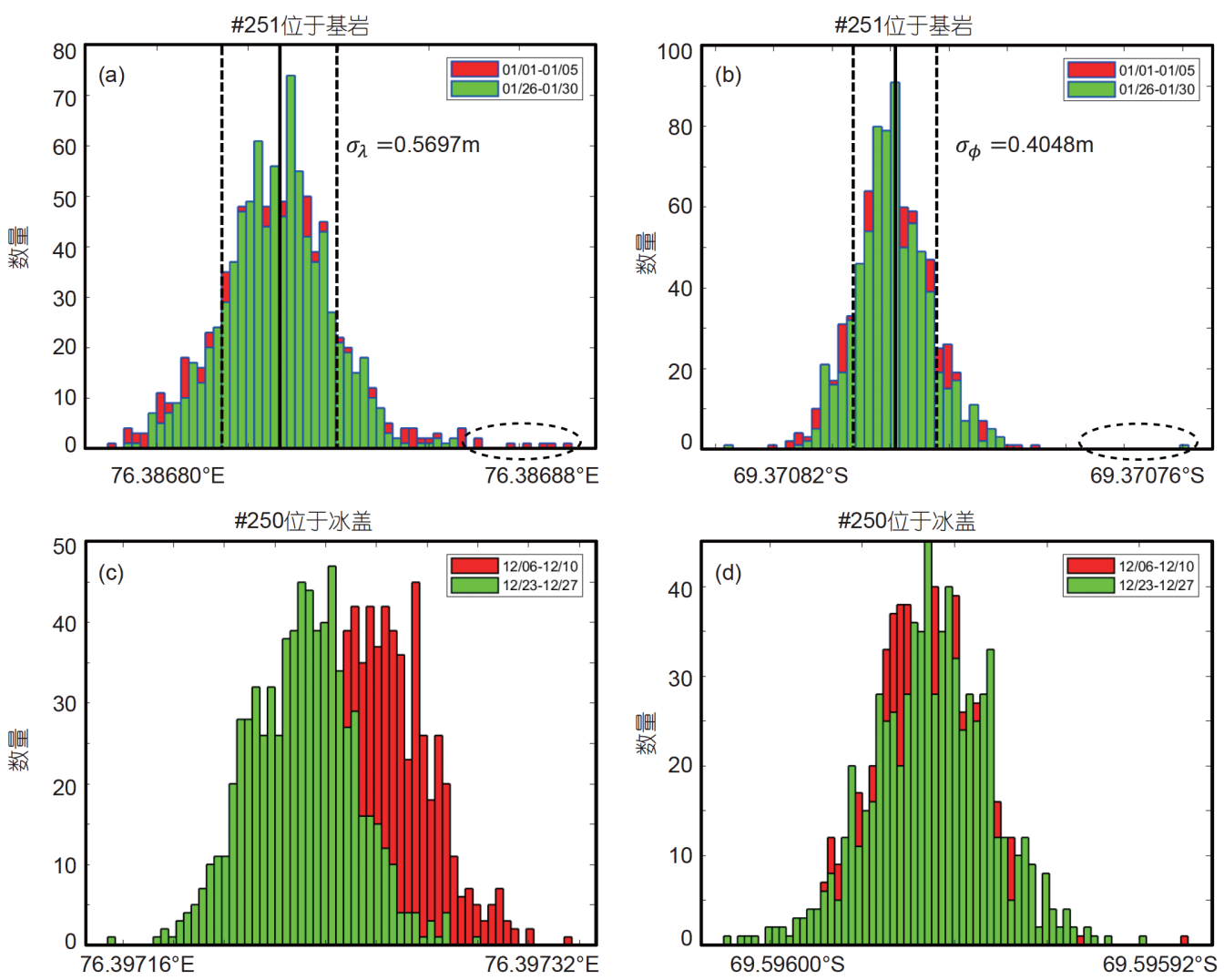

图 2 地震台站记录的原始GPS位置的直方图

2020/01/01 2020/01/30期间台站251位于露头时, 经度(a)和纬度(b)分布, 黑色虚椭圆内为偏离2.5倍标准差的异常点; 2019/12/06 2019/12/27期 间台站250位于拉斯曼丘陵冰盖处, 经度(c)和纬度(d)分布. 红色和绿色分别表示观测期间开始和结束阶段经纬度的直方分布

段，删除异常点后按公式(1)和(2)进行台站所在位置冰 盖流动方向和大小的估算, 结果如下.

\section{1 泰山站区冰盖流速}

2020年1月1日至2020年1月30日期间, 我们在泰山 站区布设了 4 台短周期地震仪器, 将这 4 台地震仪记录 的GPS位置数据进行处理, 计算的冰盖流速结果见表 1. 可见泰山站冰盖流动方向基本为 $273^{\circ}$, 观测期间冰 盖位移大小约为 $1 \mathrm{~m}$. 其流向代表了泰山站的冰盖以 $1 \mathrm{~m}$ $\mathrm{mon}^{-1}$ 的速率朝兰伯特冰川盆地移动; 此处地势平坦, 冰盖流动方向大致与等高线垂直，代表其运动主要受 控于冰盖自身重力的影响. Zhang等(2008)在距离泰山 站8km位置, 通过静态GPS将近7年(1998 2004年)的观 测数据, 追踪了泰山站的平均冰盖流动方向为 $277^{\circ}$, 冰 盖流动大小为 $17.7 \mathrm{~m} \mathrm{a}^{-1}$ (见图1a). 这与本文通过短周 期地震仪内置单模GPS位置数据追踪的冰盖运动情况 基本一致.

\section{2 拉斯曼丘陵冰盖流速}

拉斯曼丘陵冰盖流速结果见图4. 其中图4a横轴为 台站编号, 第一台地震仪位于测线最北端, 第100台地 震仪位于测线最南端; 纵轴为冰盖流动方向大小. 图中 红、绿和黑色分别为按 $5 、 6$ 和7天分段的结果; 可以发 现按6和7天分段的结果比较接近, 与按 5 天分段的结果 局部存在差异. 此外, 第 40 至 100 台计算的冰盖流动方 向连续性较好, 其流动方向基本为 $270^{\circ}$ 左右, 代表了此 处的冰盖流动整体向西偏北流动. 但是, 第1至39台的 冰盖流动方向数值在 $180^{\circ} \sim 270^{\circ}$ 范围内变化, 部分台站 差异达到了 $90^{\circ}$, 说明此局部区域冰流速比较复杂. 图 $4 \mathrm{~b}$ 为对应的冰盖流动速率结果, 可见冰盖在观测期间 (2019/12/06 2019/12/27) 冰盖流动大小基本在 $0.5 \sim 1.5 \mathrm{~m} \mathrm{mon}^{-1}$ 左右; 部分台站(如台站17 21)超过了 $1.5 \mathrm{~m} \mathrm{mon}^{-1}$, 而其对应的冰盖流动方向基本等于 $180^{\circ}$, 说明冰流速比较复杂的部分局部区域，其对应的冰盖 

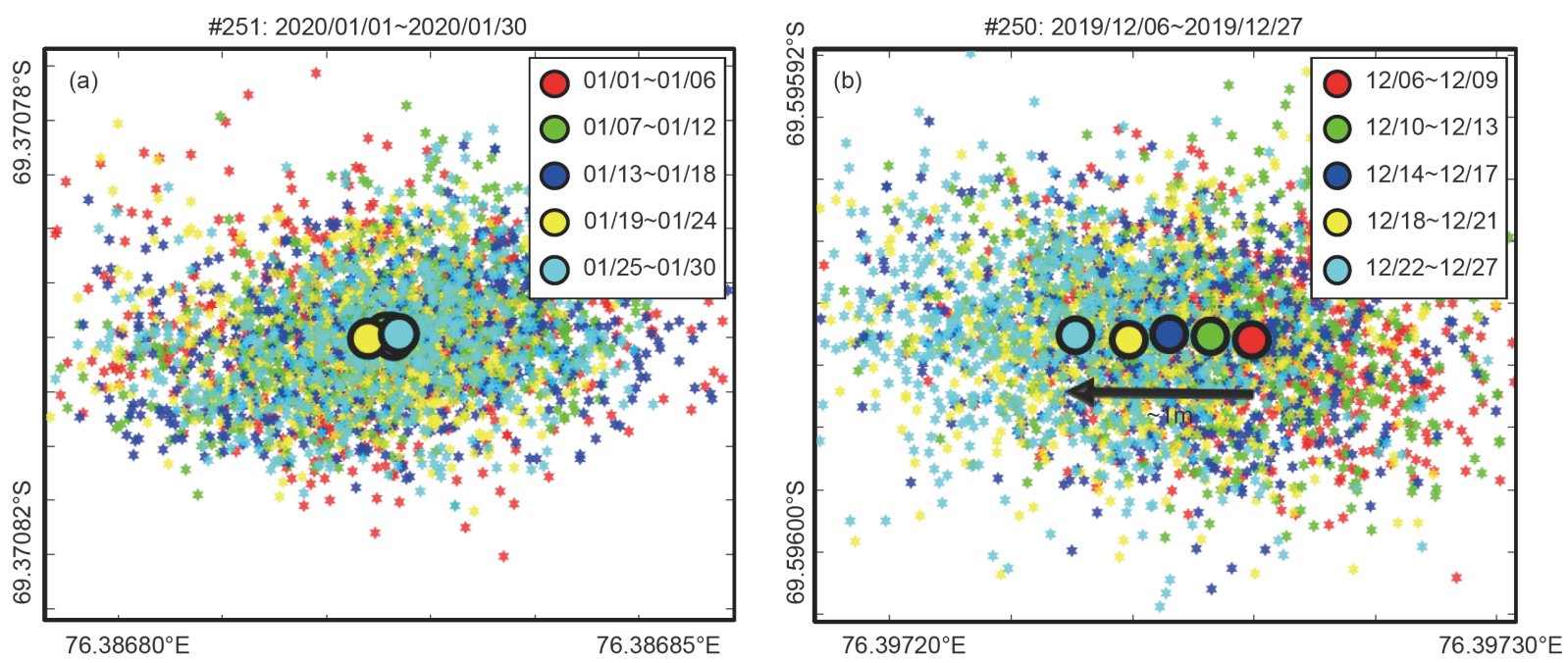

图 3 观测期间内台站所在处流动方向和位移大小(通过公式(2)估算)

(a) 当台站 251 位于中山站熊猫码头基岩, 其移动大小约为 $0.2 \mathrm{~m}$; (b) 当台站 250 位于拉斯曼丘陵冰盖, 其移动大小约为 $1 \mathrm{~m}$. 不同时间段内台站的 平均位置由红、绿、蓝、黄和青色圆圈表示

表 1 泰山站区冰盖流速结果

\begin{tabular}{|c|c|c|c|c|c|c|}
\hline \multirow{2}{*}{ 台站\# } & \multicolumn{2}{|c|}{ 日期 } & \multicolumn{2}{|c|}{ 平均位置 } & \multicolumn{2}{|c|}{ 平均速度 } \\
\hline & 开始 & 结束 & 经度 $\left({ }^{\circ} \mathrm{E}\right)$ & 纬度 $\left({ }^{\circ} \mathrm{S}\right)$ & 方向 $\left(^{\circ}\right)$ & 速率 $\left(\mathrm{m} \mathrm{mon}^{-1}\right)$ \\
\hline 288 & $2020 / 01 / 01$ & $2020 / 01 / 30$ & 76.98069 & 73.86422 & 271 & 1.05 \\
\hline 247 & $2020 / 01 / 01$ & $2020 / 01 / 30$ & 77.01110 & 73.85274 & 274 & 1.16 \\
\hline 267 & $2020 / 01 / 01$ & $2020 / 01 / 30$ & 77.01451 & 73.87502 & 273 & 1.06 \\
\hline 314 & $2020 / 01 / 01$ & $2020 / 01 / 30$ & 76.92689 & 73.86447 & 274 & 0.90 \\
\hline
\end{tabular}

移动方向和位移均存在较大差异.

图5所示为按7天时间间隔估算的拉斯曼丘陵冰流 速矢量结果, 在每个台站处红色箭头的方向代表该点 冰盖流动方向, 箭头的大小代表了冰盖位移大小. 可 见冰面高程大于 $450 \mathrm{~m}$ 的内陆冰盖, 即台站编号 40 100, 其整体向西偏北流动, 基本与等高线垂直; 代 表了此处的冰川由于重力作用, 整体向极记录冰川方 向流动. 靠近沿海的冰盖, 即台站编号 1 39, 冰面高程 约为200 400m; 其东侧为达尔克冰川, 此处冰面地形 变化剧烈, 区域冰盖流速局部变化较大, 冰舌处年均冰 流速达到 $100 \mathrm{~m} \mathrm{a}^{-1}$ (周春霞等, 2014). 在此情况下, 冰盖 流速不但受控于自身重力, 外部挤压应力对冰盖运动 的扰动不可忽略(Schoof和Hewitt, 2016).

\section{3 台站移动对地震观测的影响}

此次被动源地震观测期间, 拉斯曼丘陵冰盖1月内
流动了约 $1 \mathrm{~m}$ (图4b). 该冰流可导致台站位置约 $1 \mathrm{~m}$ 的误 差, 或可导致台站所记录的地震波传播距离的误差约 $1 \mathrm{~m}$. 冰流不均匀导致了台站间距离发生了变化, 最大 可达约 $1.86 \mathrm{~m}$ (如图4中台站\#18). 按照冰盖的横波速度 为 $1.85 \mathrm{~km} \mathrm{~s}^{-1}$ 计算, 上述误差对面波到时产生约 $1 \mathrm{~ms}$ 影 响, 可以忽略不记; 因此研究区域冰盖流动对后续面波 层析成像结果不构成影响. 对于冰流速大于几百米或 进行更长时间地震观测的情况, 冰流对台站位置和台 站间距离造成的误差有可能接近或大于本文中密集台 阵的间距 $(200 \mathrm{~m})$, 必须慎重评估冰盖流动给地震数据 处理中带来的系统性误差. 未来在南极冰盖进行短周 期被动源观测, 地震仪所到之处其冰盖流速均可通过 文中方法进行估算, 进而评估由于冰盖运动给地震学 结果带来的系统性误差; 同时也为南极大陆冰盖动力 学研究和冰盖物质平衡提供更丰富的地面GPS原位测 量结果. 

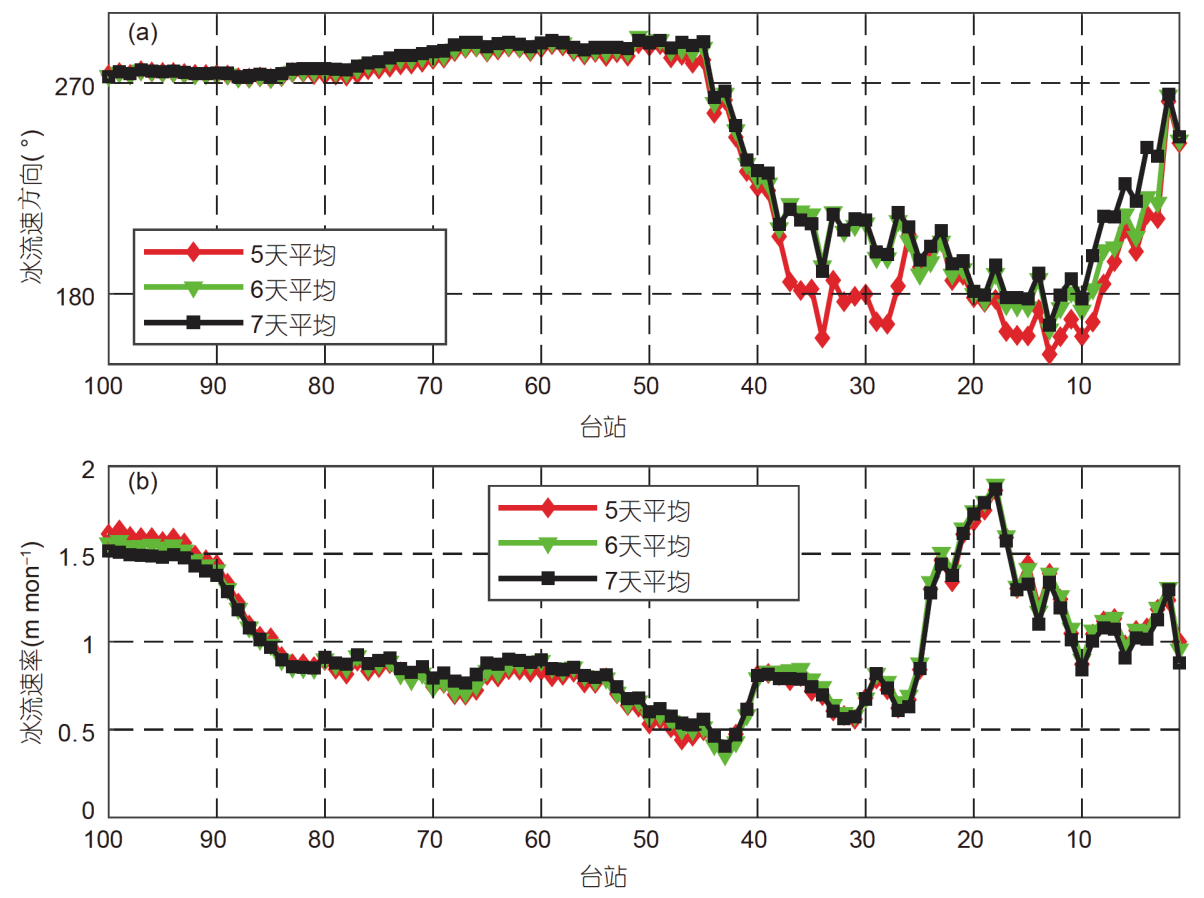

图 4 拉斯曼丘陵地震测线处对应的冰盖流动

(a) 方向; (b) 月均速率. 红色、绿色和黑色分别为按 $5 、 6$ 和 7 天时间间隔计算的结果

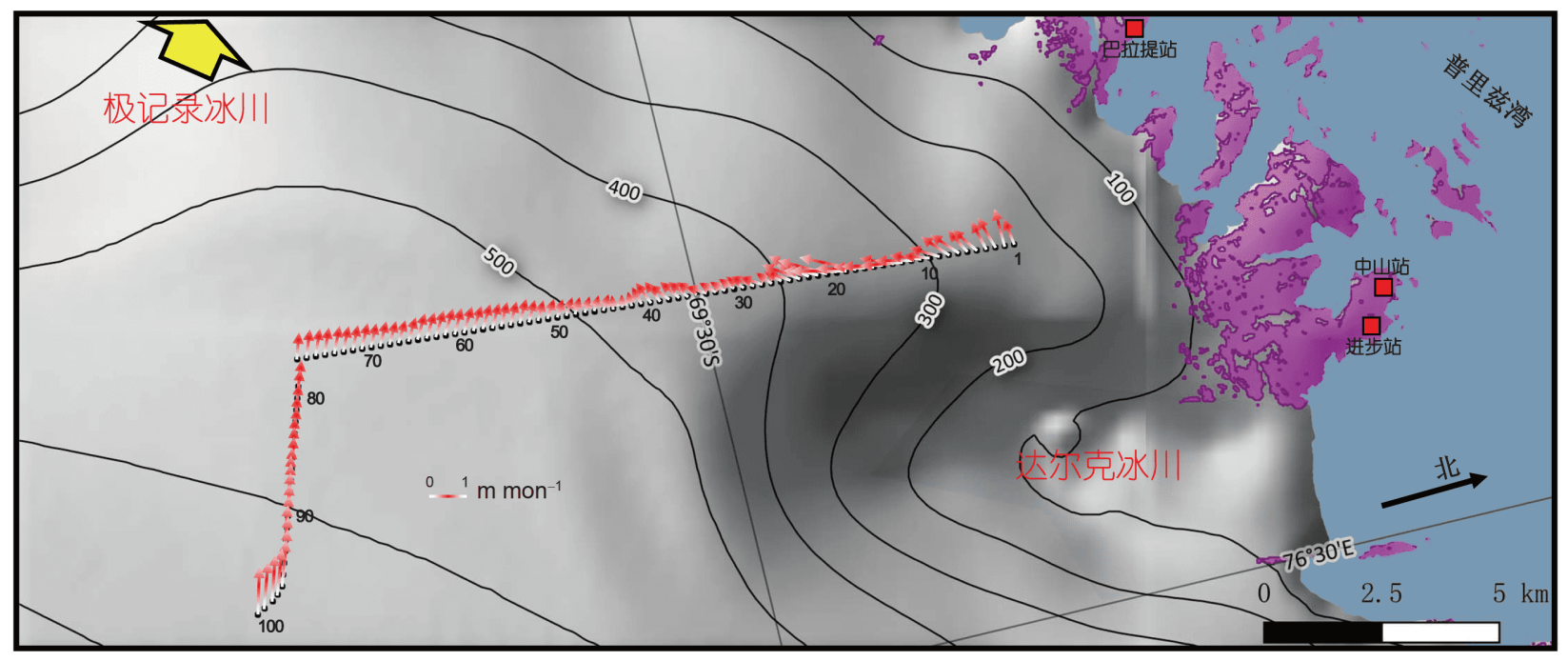

图 5 拉斯曼丘陵地震测线处冰流速矢量场

红色箭头方向和大小分别代表了冰盖流动方向和月均速率, 黄色箭头指向极记录冰川方向

\section{5 结论}

本文基于中国第36次南极考察期间被动源地震观 测时获得的导航级GPS位置信息，准确地对拉斯曼丘
陵冰盖和泰山站区冰盖运动特征进行了追踪. 拉斯曼 丘陵冰盖整体朝西流向极记录冰川，观测期间冰盖位 移大小为 $0.5 \sim 1 \mathrm{~m}$ 不等; 但靠近达尔克冰川的部分台站 受地形变化大和局部应力影响, 其冰盖流动方向变化 
大: 泰山站区冰盖整体朝西流向兰伯特冰川盆地. 泰山 站结果与通过高精度静态GPS计算结果基本一致，说 明利用短周期台站内置GPS位置信息进行冰盖运动情 况的追踪是可行的.

此次 1 个月的被动源地震观测期间, 泰山站和拉斯 曼丘陵冰盖流动了约 $1 \mathrm{~m}$, 该运动对地震观测的影响可 以忽略. 但如果冰流速大于几百米和进行长时间的观 测的话，其误差可能无法忽略. 同时考虑到降雪等的 影响，建议在南极冰盖进行短周期地震台站布设时放 置标记杆以便高效对台站进行安全回收.

致谢 感谢自然资源部极地考察办公室、中国第36次南 极科学考察固定翼飞机队成员及同济大学郝柇教授对本 次现场实验的支持; 感谢中国地质科学院安美建研究员 和同济大学乔刚副教授对本文数据处理及结果讨论中给 予的宝贵建议。

\section{参考文献}

陈军. 2016. 基于多源遥感数据的拉森冰架形态演变及表面流速估 算研究. 博士学位论文. 南京: 南京大学

王清华, 鄂栋臣, 陈春明. 2001. 中山站至A冰穹考察及沿线GPS复测 结果分析. 武汉大学学报(信息科学版), 26: 200-204

徐绍铨, 鄂栋臣, 王升定. 1988. 南极纳尔逊岛冰川运动监测. 武测科 技, 4: 30-35

周春霞, 邓方慧, 艾松涛, 王泽民, 鄂栋臣. 2014. 利用DInSAR的东南 极极记录和达尔克冰川冰流速提取与分析. 武汉大学学报(信息 科学版), 39: 940-944

周春霞，邓方慧，陈一鸣，王泽民. 2015. 利用SAR数据研究南极 Grove山地区冰流运动特征. 武汉大学学报(信息科学版), 40: $1428-1433$

An M, Wiens D, An C, Shi G, Zhao Y, Li Y. 2015. Antarctic ice velocities from GPS locations logged by seismic stations. Antarct Sci, 27: 210-222

Chylek P, Box J E, Lesins G. 2004. Global warming and the Greenland ice sheet. Clim Change, 63: 201-221

Den Ouden M A G, Reijmer C H, Pohjola V, van de Wal R S W, Oerlemans J, Boot W. 2010. Stand-alone single-frequency GPS ice velocity observations on Nordenskiöldbreen, Svalbard. Cryosphere, 4: $593-604$

Dorrer E, Hofmann W, Seufert W. 1969. Geodetic results of the Ross Ice Shelf Survey expeditions, 1962-63 and 1965-66. J Glaciol, 8: $67-90$

Fettweis X, Box J E, Agosta C, Amory C, Kittel C, Lang C, van As D, Machguth H, Gallée H. 2017. Reconstructions of the 1900-2015
Greenland ice sheet surface mass balance using the regional climate MAR model. Cryosphere, 11: 1015-1033

Frezzotti M, Capra A, Vittuari L. 1998. Comparison between glacier ice velocities inferred from GPS and sequential satellite images. Ann Glaciol, 27: 54-60

Goldstein R M, Engelhardt H, Kamb B, Frolich R M. 1993. Satellite radar interferometry for monitoring ice sheet motion: Application to an Antarctic ice stream. Science, 262: 1525-1530

Hanna E, Huybrechts P, Janssens I, Cappelen J, Steffen K, Stephens A. 2005. Runoff and mass balance of the Greenland ice sheet: 19582003. J Geophys Res, 110: D13108

Hanna E, Huybrechts P, Steffen K, Cappelen J, Huff R, Shuman C, Irvine-Fynn T, Wise S, Griffiths M. 2008. Increased runoff from melt from the Greenland Ice Sheet: A response to global warming. J Clim, 21: 331-341

Hanna E, Navarro F J, Pattyn F, Domingues C M, Fettweis X, Ivins E R, Nicholls R J, Ritz C, Smith B, Tulaczyk S, Whitehouse P L, Zwally H J. 2013. Ice-sheet mass balance and climate change. Nature, 498: 51-59

Heid T, Kääb A. 2012. Evaluation of existing image matching methods for deriving glacier surface displacements globally from optical satellite imagery. Remote Sens Environ, 118: 339-355

Hofer S, Tedstone A J, Fettweis X, Bamber J L. 2017. Decreasing cloud cover drives the recent mass loss on the Greenland Ice Sheet. Sci Adv, 3: e1700584

Holland D M, Thomas R H, De Young B, Ribergaard M H, Lyberth B. 2008. Acceleration of Jakobshavn Isbræ triggered by warm subsurface ocean waters. Nat Geosci, 1: 659-664

Joughin I, Howat I, Alley R B, Ekstrom G, Fahnestock M, Moon T, Nettles M, Truffer M, Tsai V C. 2008. Ice-front variation and tidewater behavior on Helheim and Kangerdlugssuaq Glaciers, Greenland. J Geophys Res, 113: F01004

Leeson A A, Shepherd A, Briggs K, Howat I, Fettweis X, Morlighem M, Rignot E. 2015. Supraglacial lakes on the Greenland ice sheet advance inland under warming climate. Nat Clim Change, 5: 51-55

Manson R, Coleman R, Morgan P, King M. 2000. Ice velocities of the Lambert Glacier from static GPS observations. Earth Planet Space, 52: 1031-1036

Nick F M, Luckman A, Vieli A, van der Veen C J, van As D, van de Wal R S W, Pattyn F, Hubbard A L, Floricioiu D. 2012. The response of Petermann Glacier, Greenland, to large calving events, and its future stability in the context of atmospheric and oceanic warming. J Glaciol, 58: 229-239

Oppenheimer M. 1998. Global warming and the stability of the West Antarctic Ice Sheet. Nature, 393: 325-332

Palmer S, McMillan M, Morlighem M. 2015. Subglacial lake drainage 
detected beneath the Greenland ice sheet. Nat Commun, 6: 8408

Pattyn F, Derauw D. 2002. Ice-dynamic conditions of Shirase Glacier, Antarctica, inferred from ERS SAR interferometry. J Glaciol, 48: $559-565$

Pattyn F, Ritz C, Hanna E, Asay-Davis X, DeConto R, Durand G, Favier L, Fettweis X, Goelzer H, Golledge N R, Munneke P K, Lenaerts J T M, Nowicki S, Payne A J, Robinson A, Seroussi H, Trusel L D, van den Broeke M. 2018. The Greenland and Antarctic ice sheets under $1.5^{\circ} \mathrm{C}$ global warming. Nat Clim Change, 8: $1053-$ 1061

Rignot E. 2008. Changes in West Antarctic ice stream dynamics observed with ALOS PALSAR data. Geophys Res Lett, 35: L12505

Rignot E, Mouginot J, Scheuchl B. 2011. Ice flow of the Antarctic ice sheet. Science, 333: 1427-1430

Scambos T A, Dutkiewicz M J, Wilson J C, Bindschadler R A. 1992. Application of image cross-correlation to the measurement of glacier velocity using satellite image data. Remote Sens Environ,
42: $177-186$

Schoof C, Hewitt I J. 2016. A model for polythermal ice incorporating gravity-driven moisture transport. J Fluid Mech, 797: 504-535

Shepherd A, Ivins E, Rignot E, Smith B, Van D B M, Velicogna I, Whitehouse P, Briggs K, Joughin I, Krinner G, Nowicki S. 2018. Mass balance of the Antarctic Ice Sheet from 1992 to 2017. Nature, 558: $219-222$

Straneo F, Heimbach P. 2013. North Atlantic warming and the retreat of Greenland's outlet glaciers. Nature, 504: 36-43

Trusel L D, Das S B, Osman M B, Evans M J, Smith B E, Fettweis X, McConnell J R, Noël B P Y, van den Broeke M R. 2018. Nonlinear rise in Greenland runoff in response to post-industrial Arctic warming. Nature, 564: 104-108

Zhang S K, E D C, Wang Z M, Li Y S, Jin B, Zhou C X. 2008. Ice velocity from static GPS observations along the transect from Zhongshan station to Dome A, East Antarctica. Ann Glaciol, 48: $113-118$ 